${ }^{1}$ Hospital Clínico, Departamento de Psiquiatría y Salud Mental, Universidad de Chile, Santiago, Chile.

${ }^{2}$ Facultad de Psicología, Universidad Diego Portales. Santiago, Chile. apsicóloga, Ph.D. bPsicóloga, Máster. cPsicólogo.

Fuente de apoyo financiero: FONDECYT 1130230.Licitación ID: 4127-41-LE12. I Ministerio de Salud.

Instituto Milenio para la Investigación en Depresión y

Personalidad IS130005.

Recibido el 24 de noviembre de 2014, aceptado el 26 de enero de 2015.

Correspondencia a: Dra. Graciela Rojas Avenida La Paz 1003, Recoleta, Santiago. Teléfonos: 26326933-84093801 Graciela.rojas.castillo@gmail.com

\section{Barreras de acceso a tratamiento de la depresión posparto en Centros de Atención Primaria de la Región Metropolitana: un estudio cualitativo}

\author{
GRACIELA ROJAS ${ }^{1}$, MARÍA PÍA SANTELICES ${ }^{2, a}$, \\ PABLO MARTÍNEZ ${ }^{1, c}$, ALEMKA TOMICIC ${ }^{2, a}$, MAHAIRA REINEL ${ }^{2, b}$, \\ MARCIA OLHABERRY ${ }^{2, a}$, MARIANE KRAUSE ${ }^{2, a}$
}

\section{Barriers restricting postpartum depression treatment in Chile}

Background: In Chile, postpartum depression is a prevalent and disabling condition. Universal screening is available but has not been translated into better treatment rates, suggesting the existence of access barriers. Aim: To describe access barriers to postpartum depression treatment in six primary health care clinics in Metropolitan Santiago, Chile. Material and Methods: Twenty women with postpartum depression and 18 primary health care professionals were subjected to a semi-structured interview. A qualitative methodology based on Grounded Theory was used. Results: There are user associated barriers such as lack of knowledge about the disease, a negative conceptualization and rejection of available treatment options. There are also barriers associated with poor network support and some features of the health care system such as long waiting times and lack of coordination between clinical and administrative decisions. Conclusions: Patient and provider related barriers restricting treatment of postpartum depression were identified.

(Rev Med Chile 2015; 143: 424-432)

Key words: Depression, postpartum; Health care evaluation mechanisms; Primary health care; Qualitative research.
L a depresión posparto (DPP) es frecuente en el puerperio, afecta la calidad de vida de las madres, su capacidad de vinculación con el recién nacido y el desarrollo infantil ${ }^{1,2}$.

En Chile, la prevalencia de depresión a las 8 semanas posparto asciende a $20,5 \%^{3}$ y de sintomatología depresiva entre los 2 y 3 meses posparto a $41,3 \%{ }^{4}$. A pesar de la existencia de un tratamiento efectivo ${ }^{5}$, la DPP suele no ser detectada ni tratada ${ }^{6}$.

Para promover su detección, el Ministerio de Salud (MINSAL) ${ }^{7}$, ha recomendado la aplicación de la Escala de Depresión Posparto de Edimburgo $(\mathrm{EPDS})^{8}$ en los controles de niño sano del segundo y sexto mes, en los establecimientos de atención primaria de salud (APS) .

El tamizaje de la DPP alcanza una alta cobertura y es bien aceptado por las usuarias ${ }^{6}$, sin embargo, los datos administrativos del MINSAL indican que esto no se traduce en ingreso a tratamiento, a pesar de ser una patología incorporada a las Garantías Explícitas en Salud ${ }^{10}$.

La literatura internacional ha descrito barreras para la búsqueda de ayuda y acceso a tratamiento dependientes de las madres y su entorno, de los profesionales de la salud que las atienden y del sistema de salud ${ }^{11}$. A la fecha no existe literatura nacional que explore este tema. 
El objetivo del presente estudio es describir y discutir las barreras de acceso a tratamiento de la DPP en la APS desde la perspectiva de las mujeres con DPP y de los profesionales que las atienden.

\section{Material y Método}

Este estudio formó parte de una investigación sobre uso de servicios y acceso a tratamiento de la depresión posparto en centros de APS de la Región Metropolitana, que incluyó un componente cuantitativo que detectó a 63 madres mayores de 18 años con DPP en una cohorte de 300 mujeres puérperas que comenzaran sus controles en el posparto en seis establecimientos de $\mathrm{APS}^{12}$.

El componente cualitativo se basó en la Teoría Fundamentada ${ }^{13}$, que permite generar conocimiento de tópicos poco explorados. Se empleó un diseño abierto e iterativo para la recolección y análisis de los datos, determinando su finalización por el criterio de saturación teórica, momento en que los datos no aportaron nueva información al modelo conceptual construido ${ }^{13,14}$.

Se utilizó un muestreo intencionado que permitió seleccionar a 20 madres con DPP y 18 profesionales de la salud de los centros de APS vinculados a la atención de mujeres puérperas.

Se usaron guiones de entrevista semi-estructurada para la recolección de datos (Tablas 1 y 2). Las entrevistas (en promedio $45 \mathrm{~min}$ ) fueron realizadas entre marzo y abril de 2013, las grabaciones fueron transcritas.

Se realizó un análisis descriptivo de los datos según los procedimientos de codificación abierta propuestos por la Teoría Fundamentada ${ }^{13,15}$. Se desarrollaron conceptos y categorías las que fueron organizadas en esquemas de clasificación jerárquicos. El análisis cualitativo fue asistido por el programa ATLAS.TI v7, diseñado para el manejo y procesamiento de grupos de datos textuales, que agiliza el trabajo de análisis cualitativo y su interpretación $^{16}$.

Este estudio contó con la aprobación del Comité de Ética de la Facultad de Medicina de la Universidad de Chile. Todos los participantes invitados consintieron su participación en el estudio, no hubo rechazo.

\section{Resultados}

Se entrevistaron 20 mujeres con DPP y 18 profesionales de la salud (Tabla 3 y 4 ).

\section{Tabla 1. Guión de entrevista para madres con DPP}

\begin{tabular}{|ll|}
\hline Tópico & Ejemplo \\
\hline Experiencia de la enfermedad & $\begin{array}{l}\text { ¿Me podría contar cómo fue para usted, cómo vivió el proceso de tener este } \\
\text { malestar/problema de salud? }\end{array}$ \\
\hline Proceso diagnóstico & $\begin{array}{l}\text { ¿Cómo supo que tenía este problema de salud? } \\
\text { ¿Quién se lo dijo? } \\
\text { ¿Cómo y qué le explicaron? }\end{array}$ \\
\hline Creencias respecto de la enfermedad & $\begin{array}{l}\text { ¿Qué ideas tenía/tiene respecto del origen de este problema de salud? } \\
\text { ¿Qué ideas tenía/tiene respecto de cómo se cura o se pasa este problema de } \\
\text { salud? }\end{array}$ \\
\hline Vida cotidiana y acceso a la atención & $\begin{array}{l}\text { ¿Habló usted con alguien de su familia o personas cercanas a usted sobre lo que } \\
\text { le pasaba? }\end{array}$ \\
\hline ¿Cuál fue la reacción de estas personas? (preocupación, apoyo, consejo, no \\
tomarle importancia, etc.)
\end{tabular}


Tabla 2. Guión de entrevista para profesionales de la salud en los centros de APS

\begin{tabular}{|ll|}
\hline Tópico & Ejemplo \\
\hline Experiencia con madres con DPP & $\begin{array}{l}\text { ¿Cuál ha sido su experiencia con las mujeres que presentan el diagnóstico de DPP } \\
\text { y que son derivadas a tratamiento por esta condición? }\end{array}$ \\
\hline Entrega de atención oportuna & $\begin{array}{l}\text { ¿Qué elementos relacionados con el servicio de salud en el cual usted trabaja cree } \\
\text { que facilitan o dificultan la posibilidad de entregar una atención oportuna a las } \\
\text { mujeres con DPP? }\end{array}$ \\
\hline Proceso de tamizaje & $\begin{array}{l}\text { ¿Cuál es su opinión respecto a la necesidad de un entrenamiento específico para } \\
\text { la administración de estos instrumentos (de tamizaje)? }\end{array}$ \\
\hline Indicaciones de tratamiento & $\begin{array}{l}\text { ¿Cuáles cree usted que son los elementos que facilitan u obstaculizan la } \\
\text { posibilidad de llevar a cabo indicaciones adecuadas y oportunas del tratamiento } \\
\text { de la DPP? }\end{array}$ \\
\hline Asistencia/no asistencia a tratamiento & $\begin{array}{l}\text { ¿Qué factores cree usted que son determinantes en la decisión de las mujeres con } \\
\text { diagnóstico de DPP de asistir a tratamiento? }\end{array}$ \\
\hline
\end{tabular}

Tabla 3. Caracterización de madres con DPP

\begin{tabular}{|c|c|}
\hline & $n=20$ \\
\hline Edad (años) & $28,4(6,5)$ \\
\hline \multicolumn{2}{|l|}{ Estado civil } \\
\hline Soltera & $13(65)$ \\
\hline Casada & $3 \quad(5)$ \\
\hline Conviviente & 1 (15) \\
\hline Separada o divorciada & $3(15)$ \\
\hline \multicolumn{2}{|l|}{ Escolaridad } \\
\hline $0-8$ años & $3(15)$ \\
\hline 9-12 años & $14(70)$ \\
\hline Más de 12 años & $3(15)$ \\
\hline \multicolumn{2}{|l|}{ Ocupación principal } \\
\hline Dueña de casa & $14(70)$ \\
\hline Trabajadora & $6(30)$ \\
\hline \multicolumn{2}{|l|}{ Vive con } \\
\hline Pareja y otros & $5 \quad(25)$ \\
\hline Padres y otros & $8(40)$ \\
\hline Sola con hijos & $3(15)$ \\
\hline Otros & $4(20)$ \\
\hline Número de hijos & $2,2(0,9)$ \\
\hline Diagnóstico previo de depresión & $13(65)$ \\
\hline Puntaje EPDS & $17,2(4,6)$ \\
\hline
\end{tabular}

Los datos son $\mathrm{n}(\%)$ o promedio (SD).
Tabla 4. Caracterización de profesionales participantes

\begin{tabular}{|lc|}
\hline Caracterización profesionales & $\mathbf{n}=\mathbf{1 8}$ \\
\hline Sexo (mujer) & $16(88,9)$ \\
Profesión & \\
Médico(a) & $4(22,2)$ \\
Matrona & $5(27,8)$ \\
Enfermera & $4(22,2)$ \\
Psicólogo(a) & $5(27,8)$ \\
\hline
\end{tabular}

Los datos son $\mathrm{n}(\%)$.

\section{Temas emergentes}

Siete categorías relevantes para el estudio, se describen a continuación.

\section{Representación de la DPP}

Las mujeres con DPP relacionan la enfermedad con debilidad, flojera, embarazo no deseado, miedo al rechazo por estar embarazada, riesgo social, conflicto con la pareja, dificultades con los hijos, muerte de algún familiar u otro significativo, violencia intrafamiliar, historia infantil de abandono $\mathrm{y}$ antecedentes de salud mental.

Ellas creen que durante el embarazo no se puede estar triste, anhelando felicidad y tranquilidad. Algunas mencionan emociones negativas y conflictos con el padre del hijo(a), destacándose 
episodios de abandono, violencia y maltrato. En el posparto, las emociones negativas son vividas con culpa y significadas como ser una "mala madre". Ellas evidencian un deterioro en la relación con familiares y otros significativos, quienes se manifiestan incrédulos del malestar de la entrevistada. Se agrega la vivencia de una relación negativa consigo misma y con el cuerpo, sensación de dificultad en el apego con la guagua, devaluación y despreocupación de la imagen corporal, y malnutrición. Refieren que la DPP interfiere en la vida cotidiana, sobre todo en las tareas diarias relacionadas con la crianza.

Respecto al tratamiento, no hay consenso entre las afectadas. Algunas plantean que la DPP requiere de tratamiento para aliviar el malestar, mientras otras creen en una mejoría espontánea (Figura 1).

\section{La solicitud de ayuda}

Las madres deprimidas reconocen como barreras para pedir ayuda, la dificultad de definir como enfermedad su malestar psicológico, la falta de redes de apoyo y el miedo a ser catalogada de "loca", la creencia de que la depresión se supera con fuerza de voluntad, lo que conlleva a una desestimación de los beneficios que puede tener el tratamiento médico y psicológico. Junto con esto, presentan creencias negativas asociadas a la depresión postparto y la maternidad entre las que predominan el miedo al rechazo de sus familiares, miedo a que le quiten el bebé, vergüenza de reconocer el rechazo que sienten hacia el bebé, y falta de confianza hacia el personal de los consultorios.

Los profesionales entrevistados identifican como barreras de las madres para pedir ayuda, el temor a expresar emociones negativas y al igual que ellas, el temor a que les quiten el bebé. Asimismo, refieren que el subdiagnóstico de la DPP en el consultorio es otra importante barrera.

Entre los facilitadores para la búsqueda de ayuda, las mujeres mencionan (a) en relación al consultorio: confianza en el personal, satisfacción con

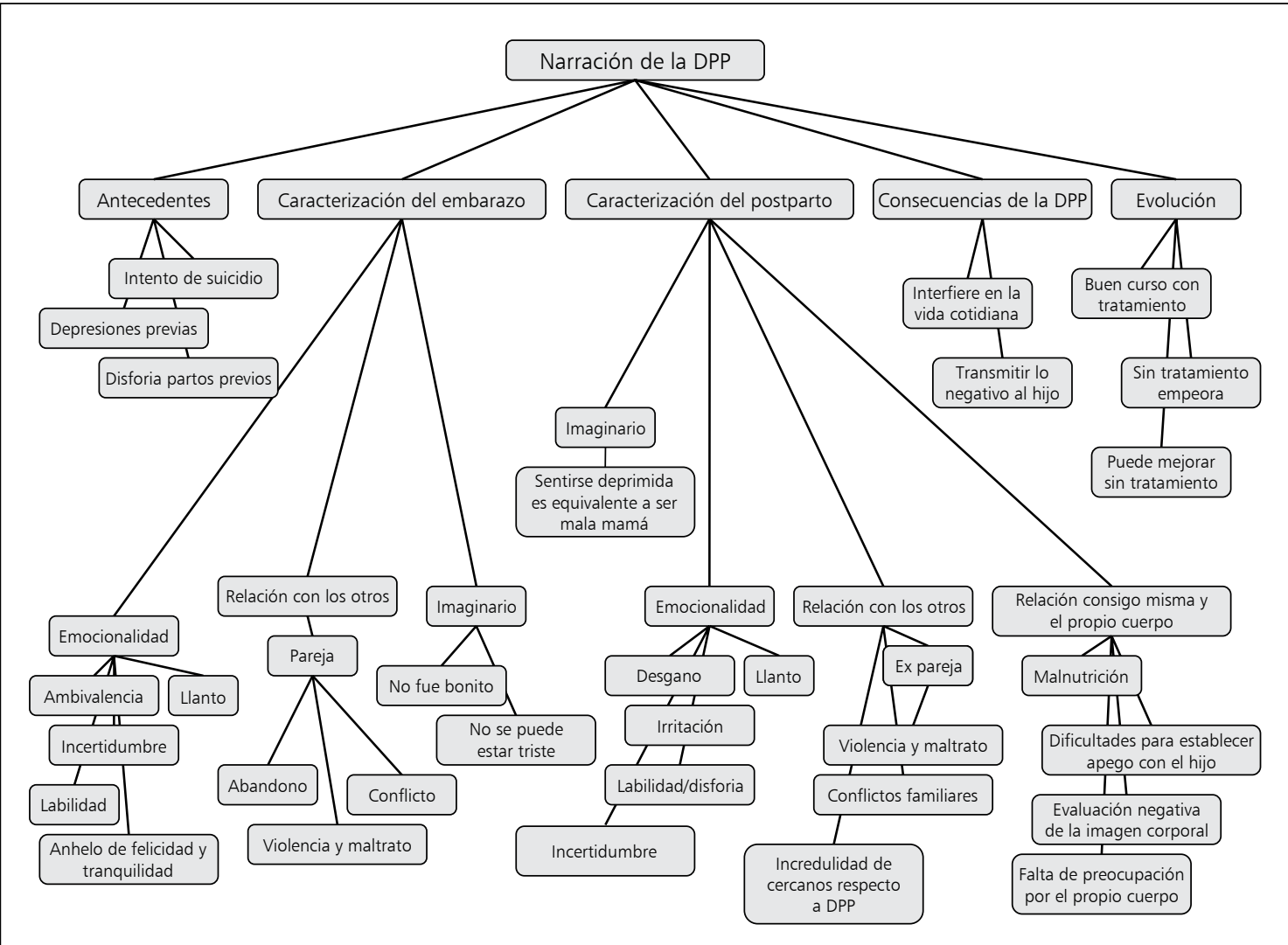

Figura 1. Esquema de clasificación jerárquica de la categoría "Representación de la DPP". 
la atención, facilidades en el acceso a las consultas y contención al momento de recibir diagnóstico; (b) en relación a la red de apoyo: retroalimentación e indicaciones de otros significativos respecto de su ánimo, y que un familiar cercano le sugiera pedir ayuda; y (c) relacionados con la DPP: sensación de malestar psicológico, detección de necesidad de ayuda facilitada por experiencias previas de tratamiento, y motivación de cambio por el bienestar de su hijo(a) (Figura 2).

\section{Tamizaje y diagnóstico}

La comunicación del diagnóstico es considerada un aspecto central para la adherencia a tratamiento tanto por las mujeres como por los profesionales. Para éstos constituye una posibilidad de intervención, pues libera a las mujeres de prejuicios negativos sobre la depresión y la maternidad.

Los profesionales declaran que el tamizaje es aplicado por matrón(a) o enfermero(a), y confirmado por médico(a) o psicólogo(a). Las madres identifican adicionalmente a otros profesionales, como asistentes sociales y médicos(as) especialistas.

Los profesionales entrevistados identifican el EPDS como un instrumento útil para el diagnóstico y algunos agregan los criterios diagnósticos CIE-10 junto con la observación del estado de la díada y calidad del vínculo madre-hijo(a).

\section{La atención}

Los profesionales mencionan que la primera atención suele ser realizada por matrón(a) o, en su defecto, enfermero(a). Se reconoce como positivo la experiencia y acompañamiento hecho por matrón(a) durante el embarazo.

Los profesionales indican que un EPDS positivo/alterado y la presencia de suicidabilidad instalan la sospecha de DPP.

Tras la sospecha, la madre es derivada a médico(a) o psicólogo(a) en el mismo consultorio. En

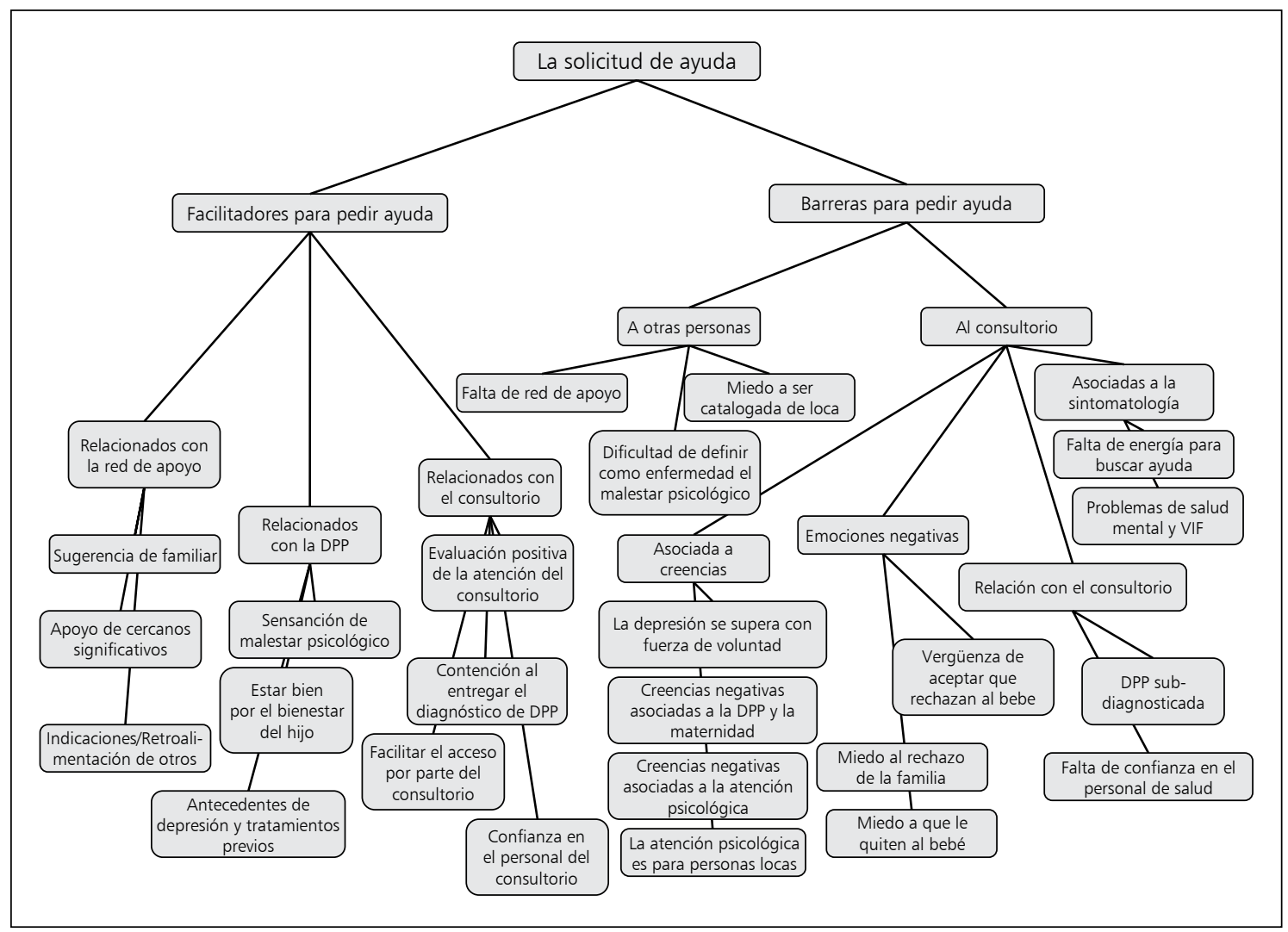

Figura 2. Esquema de clasificación jerárquica de la categoría "La solicitud de ayuda". 
casos graves, existiría la posibilidad de derivación.

La estrategia búsqueda activa de la madre por parte del consultorio, mencionada por profesionales, no siempre sería efectiva y demandaría mayores recursos.

\section{El tratamiento}

Los entrevistados identifican a médico(a), psiquiatra, psicólogo(a), matrón(a), asistente social y paramédico(a) involucrados en el tratamiento.

Respecto al tipo de intervención abundan las menciones al tratamiento farmacológico y la psicoterapia.

Los profesionales refieren el programa Chile Crece Contigo y el protocolo AUGE para depresión, las madres deprimidas nombran exámenes de sangre, medicamentos tranquilizantes e inductores de sueño y Flores de Bach.

\section{Razones para no adherencia al tratamiento}

Asociadas con (a) el consultorio: escaso tiem- po de atención, falta de precisión en el diagnóstico y la derivación, escaso reconocimiento de condiciones de riesgo, falta de un programa de salud mental, dificultad de establecer un vínculo positivo y de confianza con el personal de salud; (b) el tratamiento: la experimentación de alivio sintomático, miedo al efecto de los fármacos en los hijos, percepción de ineficacia del tratamiento, especialmente referido a la escasa percepción de resultados concretos en la psicoterapia ("sólo conversar"); (c) las mujeres: demandas de la vida cotidiana intensificadas por condiciones de riesgo social, sobrecarga de trabajo, falta de apoyo familiar y motivación, y el tiempo invertido en labores domésticas no remuneradas (Figura 3).

\section{Red de apoyo}

Las redes de apoyo son similares en todas las madres deprimidas: escasas y circunscritas a figuras significativas de la familia nuclear, generalmente de sexo femenino (hermanas, madre), que

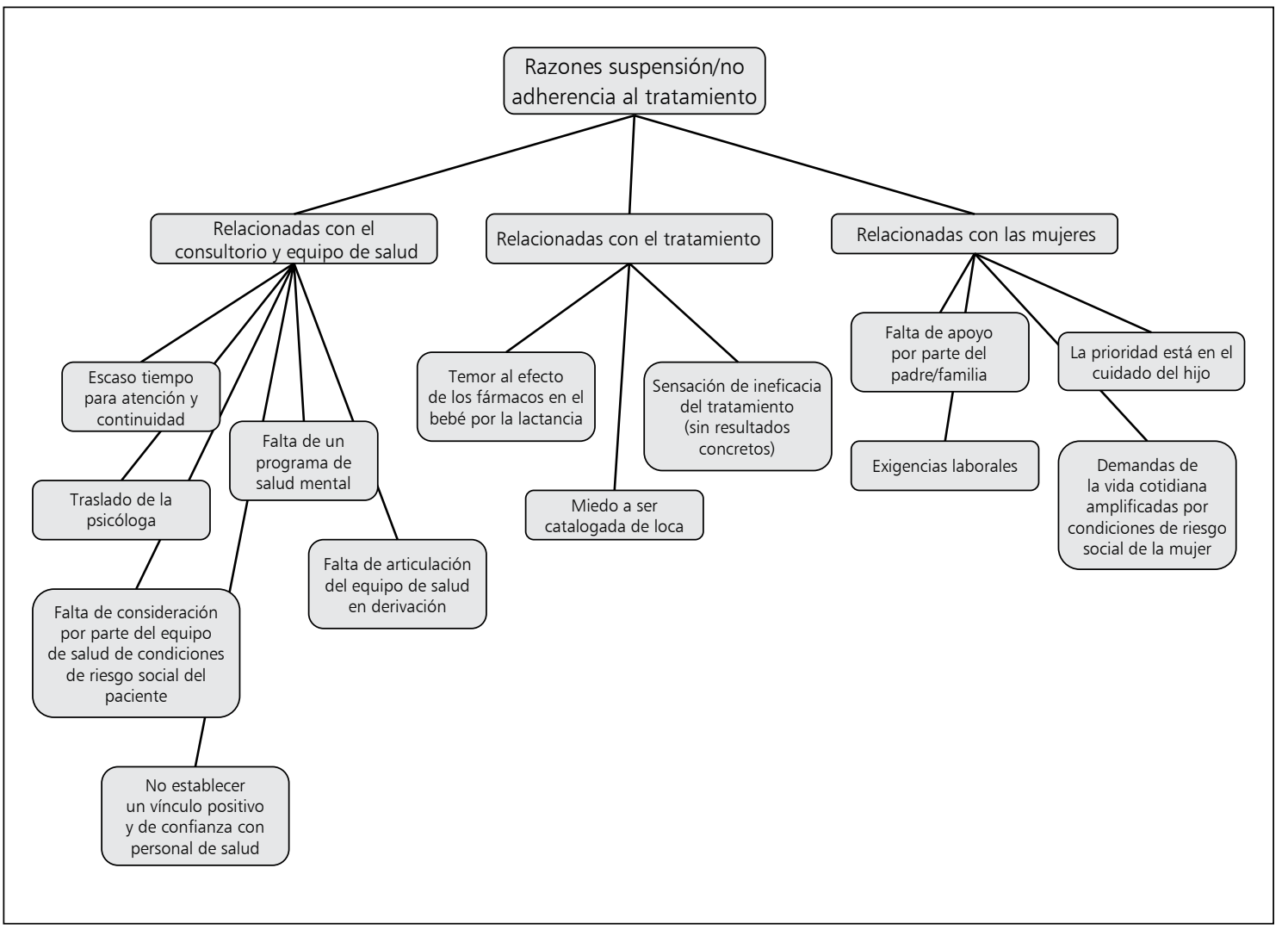

Figura 3. Esquema de clasificación jerárquica de la categoría "Razones para no adherencia al tratamiento". 
prestan auxilio en la provisión de cuidado, apoyo psicológico, emocional e informativo.

\section{Discusión}

Este es el primer estudio en Chile que identifica barreras y facilitadores para el acceso a tratamiento de la DPP desde la perspectiva de madres que padecen esta patología y de profesionales de la salud.

Las mujeres con DPP entrevistadas reconocen sufrir alteraciones emocionales y enfrentar problemas psicosociales durante el período perinatal y que sus antecedentes previos de salud mental pueden ser posibles causas de su malestar.

Sin embargo, si bien las madres son capaces de detectar el malestar anímico, tienen dificultades para interpretarlo como una enfermedad, lo que se relaciona a creencias en torno a la maternidad y la patología mental y temor a ser rotuladas como locas, malas madres o a perder la tuición del bebé.

Los participantes destacan que la comunicación clara de la sospecha es fundamental para aceptar y adherir el proceso terapéutico, destacando que un vínculo de confianza y mayor disponibilidad de horas asistenciales facilitarían el acceso a tratamiento.

La asistencia de las mujeres a los establecimientos de APS ha de conciliarse con todas las exigencias de su vida cotidiana intensificadas por condiciones de riesgo social, suponiendo la movilización de recursos a veces no disponibles. Lo anterior, junto a la percepción de escasa flexibilidad en la asignación de horas asistenciales y tiempos extensos de espera, se convierte en barreras de acceso.

Finalmente, las preocupaciones por posibles efectos sobre la salud del bebé son motivo de rechazo del tratamiento farmacológico, en tanto que la percepción de ineficacia lo es para las intervenciones psicológicas.

Las causas atribuidas por las madres a la DPP son congruentes con los factores de riesgo descritos para la patología según la literatura nacional ${ }^{17-19}$ e internacional ${ }^{20,21}$, específicamente los problemas emocionales e interpersonales, principalmente las relaciones de pareja, durante el embarazo.

Sobre las dificultades de las madres para aceptar la DPP, la evidencia internacional reporta que 90\% de éstas reconocen un malestar, pero sólo un tercio cree estar enferma ${ }^{22}$. Una posible expli- cación al fenómeno pudiera ser el curso crónico de la depresión y la presencia del trastorno al interior de la familia que tiende a normalizar la sintomatología.

Estudios cualitativos extranjeros han descrito que familiares y amigos podrían obstaculizar la búsqueda de ayuda normalizando los síntomas o manifestando una escasa comprensión de la $\mathrm{DPP}^{11,23}$. En conjunto con lo señalado, se ha referido que un ideal de maternidad exigente, compartido por las mujeres y su entorno, genera temor a no cumplir con las expectativas y reticencia a aceptar emociones negativas no validadas socialmente ${ }^{24-26}$.

La importancia atribuida a la comunicación adecuada de la sospecha de DPP es respaldada por investigaciones recientes que sugieren la necesidad de entrenamiento del personal de salud para efectuar un proceso de detección integrado a la práctica clínica, que contemple la psicoeducación y acompañamiento a mujeres con sospecha de $\mathrm{DPP}^{27,28}$.

La evidencia cualitativa en países desarrollados destaca la relevancia que el personal de salud establezca vínculos positivos para la apertura de problemas emocionales, puntualizando la ventaja que supone la continuidad en los cuidados de la salud materno-infantiles para construir este vínculo ${ }^{24,25}$.

Las barreras estructurales de accesibilidad ${ }^{29,30}$ y la conciliación de exigencias contrapuestas ${ }^{29,31,32}$ han sido documentadas principalmente en madres vulnerables socialmente, según la literatura internacional. La disponibilidad de horas y tiempos de espera extensos han sido referidas como características del sistema de salud que obstaculizan el acceso y la adherencia al tratamiento ${ }^{11,24}$. Los estudios internacionales que han explorado la aceptabilidad del tratamiento subrayan el temor de las madres a los efectos de los fármacos y una inclinación mucho más favorable hacia la psicoterapia individual ${ }^{24,33}$, aunque con percepción de eficacia moderada ${ }^{25}$.

La atribución causal psicosocial de la DPP que realizan las madres parece jugar un importante rol en la escasa adherencia a las intervenciones desde el sistema de salud ${ }^{34}$, lo que apoyaría la alternativa de tratamientos psicosociales con apoyo a la familia y/o llevados a cabo en el domicilio ${ }^{35,36}$.

Otro aspecto a destacar es la relación del binomio madre-hijo(a) al momento de ofrecer alternativas de tratamiento. Los estudios muestran múltiples efectos negativos de la DPP en la 
salud mental y el desarrollo infantil ${ }^{37,38}$ y que intervenir sólo en la depresión materna no asegura mejoras en la calidad vincular madre-bebé ${ }^{39,40}$. Respaldando esta propuesta, algunos estudios internacionales muestran una mayor satisfacción con los tratamientos en mujeres con DPP cuando estos consideran atención multidisciplinaria que incluye al padre y al bebé ${ }^{41}$.

El número reducido de participantes, la homogeneidad de éstos, y la acotación del estudio a comunas urbanas de la Región Metropolitana, son importantes limitaciones para la generalización de los resultados. Sin embargo, los resultados descritos pueden orientar a la búsqueda de estrategias para facilitar el acceso a tratamiento de las mujeres que sufren DPP.

En este sentido, se recomienda evaluar la posibilidad de:

1. Educación sobre la DPP para las madres y sus familias, que considere distintos medios informativos para facilitar la identificación de la patología, contrarrestar los estigmas sociales e informar sobre las diferentes intervenciones.

2. Ajustes sistémicos en los establecimientos de APS para facilitar la detección -incluso durante el embarazo- de factores de riesgo para DPP, garantizar el acceso a tratamiento: mejorar la disponibilidad de horas según criterios clínicos, facilitar el transporte hacia el consultorio o la asistencia de madres con menores de edad, integrar el tamizaje a la práctica clínica e iniciarlo en el embarazo y velar por la continuidad en los cuidados materno-infantiles.

3. Entrenar al personal de salud en: i) comunicación efectiva para establecer vínculos terapéuticos positivos con las madres deprimidas; y ii) alternativas de tratamiento, considerando la calidad vincular madre-bebé, y las características clínicas y de personalidad de quienes presentan la patología.

Agradecimientos: A los centros participantes.

\section{Referencias}

1. Rojas G, Fritsch R, Solís J, González M, Guajardo V, Araya R. Calidad de vida de mujeres deprimidas en el posparto. Rev Med Chile 2006; 134 (6): 713-20.

2. Parsons CE, Young KS, Rochat TJ, Kringelbach ML, Stein A. Postnatal depression and its effects on child development: a review of evidence from low-and middle-income countries. Br Med Bull 2012; 101 (1): 57-79. doi: 10.1093/bmb/ldr047.

3. Alvarado R, Rojas M, Monardes J, Neves E, Olea E, Perucca E, et al. Cuadros depresivos en el posparto y variables asociadas en una cohorte de 125 mujeres embarazadas. Rev Psiquiatr 1992; 3-4: 1168-76.

4. Jadresic E, Araya R. Prevalencia de depresión posparto y factores asociados en Santiago, Chile. Rev Med Chile 1995; 123: 694-99.

5. Rojas G, Fritsch R, Solís J, Jadresic E, Castillo C, González $\mathrm{M}$, et al. Treatment of postnatal depression in low-income mothers in primary-care clinics in Santiago, Chile: a randomised controlled trial. Lancet 2007; 370 (9599): 1629-37.

6. Castañón C, Pinto J. Mejorando la pesquisa de depresión posparto a través de un instrumento de tamizaje, la escala de depresión posparto Edimburgo. Rev Med Chile 2008; 136: 851-8.

7. MINSAL. Guía Clínica Depresión en personas de 15 años y más. Chile: Ministerio de Salud, 2013.

8. Jadresic E, Araya R, Jara C. Validation of the Edinburgh Postnatal Depression Scale (EPDS) in Chilean postpartum women. J Psychosom Obstet Gynaecol 1995; 16: 187-91.

9. MINSAL. Manual para el apoyo y seguimiento del desarrollo psicosocial de los niños y niñas de 0 a 6 años. Chile: Ministerio de Salud, 2008.

10. MINSAL. Segundo Régimen de Garantías Explícitas en Salud. Chile: Ministerio de Salud, 2006.

11. Dennis CL, Chung-Lee L. Postpartum depression help-seeking barriers and maternal treatment preferences: a qualitative systematic review. Birth 2006; 33 (4): 323-31.

12. MINSAL. Estudio sobre análisis de la situación de uso de servicios y acceso a tratamiento de la depresión post parto en centros APS de la Región Metropolitana. Chile: Unidad Chile Crece Contigo-Ministerio de Salud, 2013.

13. Strauss AL, Corbin J. Basics of qualitative research. Newbury Park-London-New Delhi: Sage, 1991.

14. Flick U. Introducción a la Investigación Cualitativa. Madrid: Ediciones Morata, 2007.

15. Charmaz K. Constructing grounded theory. A practical guide through qualitative analysis. Los Angeles: Sage publications, 2006.

16. Muños J. Análisis cualitativos de datos textuales con Atlas.ti 5. Creative Commons, 2005. Disponible en: http://www.ucpr.edu.co/centros/investigaciones/Atlas5. pdf. (Consulta: 13 de junio de 2013).

17. Hasbún J, Risco L, Jadresic E, Galleguillo T, González M, Garay S. Depresión posparto: prevalencia y factores de riesgo. Rev Chil Obstet Ginecol 1999; 64 (6): 466-70. 
18. Jadresic E, Jara C, Araya R. Depresión en el embarazo y el puerperio: estudio de factores de riesgo. Acta psiquiat psicol Am lat 1992; 39 (1): 63-74.

19. Poo AM, Espejo C, Godoy C, Gualda M, Hernández T, Pérez C. Prevalencia y factores de riesgo asociados a la depresión posparto en puérperas de un Centro de Atención Primaria en el Sur de Chile. Rev Med Chile 2008; 136 (1): 44-52.

20. Cantwell R, Smith S. Prediction and prevention of perinatal mental illness. Psychiatry 2006; 5 (1): 15-21.

21. Robertson E, Grace S, Wallington T, Stewart DE. Antenatal risk factors for postpartum depression: a synthesis of recent literature. Gen Hos Psychiatry 2004; 26 (4): 289-95.

22. Whitton A, Warner R, Appleby L. The pathway to care in post-natal depression: women's attitudes to post-natal depression and its treatment. Br J Gen Pract 1996; 46 (408): 427-8.

23. Sword W, Busser D, Ganann R, McMillan T, Swinton M. Women's care-seeking experiences after referral for postpartum depression. Qual Health Res 2008; 18 (9): 1161-73.

24. Bilszta J, Ericksen J, Buist A, Milgrom J. Women's experience of postnatal depression -beliefs and attitudes as barriers to care. AJAN 2010; 27 (3): 44-54.

25. Chew-Graham C, Sharp D, Chamberlain E, Folkes L, Turner K. Disclosure of symptoms of postnatal depression, the perspectives of health professionals and women: a qualitative study. BMC Fam Pract 2009; 10: 7. doi: 10.1186/1471-2296-10-7.

26. Edge D, MacKian SC. Ethnicity and mental health encounters in primary care: help-seeking and help-giving for perinatal depression among Black Caribbean women in the UK. Ethn Health 2010; 15 (1): 93-111.

27. Glavin K, Ellefsen B, Erdal B. Norwegian public health nurses' experience using a screening protocol for postpartum depression. PHN 2010; 27 (3): 255-62.

28. Yawn B, Olson A, Bertram S, Pace W, Wollan P, Dietrich A. Postpartum depression: screening, diagnosis and management programs 2000 through 2010. Depress Res Treat 2012; 2012: 363964 . doi: 10.1155/2012/363964.

29. Callister LC, Beckstrand RL, Corbett C. Postpartum depression and help-seeking behaviors in immigrant Hispanic women. J Obstet Gynecol Neonatal Nurs 2011; 40 (4): 440-9.
30. O'Mahen H, Flynn H. Preferences and perceived barriers to treatment for depression during the perinatal period. J Womens Health 2008; 17 (8): 1301-9.

31. O’Mahony J, Donnelly T. Immigrant and refugee women's post-partum depression help-seeking experiences and access to care: a review and analysis of the literature. J Psychiatr Men Health Nurs 2010; 17 (10): 917-28.

32. Kurtz-Landy C, Sword W, Ciliska D. Urban women's socioeconomic status, health service needs and utilization in the four weeks after postpartum hospital discharge: findings of a Canadian cross-sectional survey. BMC Health Serv Res 2008; 8: 203. doi: 10.1186/1472-6963-8-203.

33. Cabrol H, Teissedre F, Armitage J, Danel M, Walburg V. Acceptability of psychotherapy and antidepressants for postnatal depression among newly delivered mothers. J Reprod Infant Psychol 2004; 22 (1): 5-12.

34. Oates M, Cox JL, Neema S, Asten P, Glangeaud-Freudenthal N, Figuereido B, et al. Postnatal depression across countries and cultures: a qualitative study. Br J Psychiatry 2004; 184: s10-s16.

35. Goodman JH. Women's attitudes, preferences, and perceived barriers to treatment for perinatal depression. Birth 2009; 36 (1): 60-9.

36. Sharon-Segre L, Stasik SM, O'Hara MW, Arndt S. Listening visits: an evaluation of the effectiveness and acceptability of a home-based depression treatment. Psychother Res 2010; 20 (6): 712-21.

37. Boyd R, Zayas L, McKee M. Mother-infant interaction, life events, and prenatal and postpartum depressive symptoms among minority women in primary care. Matern Child Health J 2006; 10: 139-48. doi: 10.1007/ s10995-005-0042-2.

38. Downey G, Coyne JC. Children of depressed parents: an integrative view. Psychol Bull 1990; 108: 50-76.

39. Murray L, Cooper P, Wilson A, Romaniuk H. Controlled trial of the short and long-term effect of psychological treatment of post-partum depression 2. Impact on the mother-child relationship and child outcome. Br J Psychiatry 2003; 182: 420-7.

40. Puckering C. Mind the gap! Helping the children of mothers with postnatal depression. Child Care Health Dev 2005; 31: 7-9.

41. Boath E, Bradley E, Henshaw C. User's views of two alternative approaches to the treatment of postnatal depression. J Reprod Infant Psychol 2004; 22: 13-24. 